\title{
SISTEMA DE CONTROLE DA PRODUÇÃO SIDERURGICA BASEADO EM TRANSFERÊNCIA DO CONHECIMENTO*
}

\author{
Leonardo Matsumota ${ }^{1}$ \\ Leonardo Silva Costa ${ }^{2}$ \\ Luiz Claudio Costa ${ }^{3}$ \\ Nilson Magalhães Bueno 4 \\ Oduvaldo Vendrametto 5
}

\section{Resumo}

Este trabalho aborda um estudo de caso sobre a transferência do conhecimento siderúrgico de colaboradores da empresa para o sistema que realiza o controle de produção da Usina de Cubatão. Este processo de transferência foi realizado em etapas até a implantação do sistema que registra os principais eventos realizados pelas máquinas operacionais. Estes registros são armazenados em banco de dados e com o uso de ferramentas, podem ser apresentados em relatórios e gráficos. Com isso, a empresa possui um sistema de produção muito confiável, por ter condição de analisar e diagnosticar cada etapa do processo de produção e acompanhar com precisão as falhas e acertos operacionais. Quando há estabilidade no processo e todos os eventos acontecem de acordo com a condição normal de operação, o sistema possibilita um controle aprimorado no consumo de gás e da produtividade. $O$ método utilizado é baseado em um projeto de experimentos que examina a influência dos principais parâmetros de controle no processo de produção industrial.

Palavras-chave: PIMS; Sistema de controle; Automação; Siderurgia.

\section{CONTROL SYSTEM PRODUCTION OF STEEL BASED ON KNOWLEDGE TRANSFER}

\section{Abstract}

This paper discusses a case study on the transfer of knowledge of the steel company employees to the system which controls production of Cubatão mill. This transfer process was done in stages until the implementation of the system that records the major events held by operating machinery. These records are stored in the database and with the use of tools, can be presented in reports and graphs. With this, the company has a very reliable production system, by having to analyze and diagnose the condition of each stage of the production process and accurately track the flaws and operational successes. When there is stability in the process and all events happen according to the normal operating condition, the system enables enhanced in gas consumption and productivity control. The method used is based on a design of experiments examining the influence of the main control parameters in the industrial production process.

Keywords: PIMS; Control system; Automation; Steel mill.

1 Engenharia de Produção, Mestrando, Analista de Automação, Engenharia de Manutenção, Usiminas, Cubatão, SP, Brasil.

2 Automação Industrial e Controle de Processos, Pós-Graduado, Analista de Automação, Engenharia de Manutenção, Usiminas, Cubatão, SP, Brasil.

3 Engenharia Elétrica, Mestrado, Gerente de Engenharia Industrial, Engenharia Industrial, Usiminas, Cubatão, SP, Brasil.

4 Engenharia Elétrica, Mestrado, Coordenador do Curso Superior de Tecnologia, UNIP, Santos, SP, Brasil.

5 Engenharia de Produção, Doutorado, Coordenador, UNIP, São Paulo, SP, Brasil.

* Contribuição técnica ao $18^{\circ}$ Seminário de Automação e TI Industrial, 23 a 26 de setembro de 2014, São Paulo, SP, Brasil. 


\section{INTRODUÇÃO}

O conhecimento é um bem valioso e reconhecidamente importante nas organizações por contribuir em aspectos estratégicos como processo de inovação, manutenção e melhoria continua da empresa. De acordo com Tarapanoff [1], as mudanças que vêm ocorrendo nas organizações atualmente convergem para a quebra de um paradigma histórico e, por meio dele, entramos na era sociedade da informação e do conhecimento.

Segundo Nonaka e Takeuchi [2] o conhecimento humano pode ser categorizado em dois tipos: tácito e explícito. Conhecimento tácito é aquele que está na mente das pessoas, possuindo forte ligação com a experiência de cada indivíduo, difícil de ser compartilhado e dependente da história de vida de cada um, de seus valores e modelos mentais; ou seja, seria o conhecimento subjetivo. Já o conhecimento explícito é codificado e pode ser encontrado em documentos e projetos, sendo materializado no próprio produto, ou seja, o conhecimento objetivo.

De acordo com Tarapanoff [1], a criação de conhecimento organizacional pode ser definida como a capacidade que uma instituição tem de criar conhecimento, disseminá-lo na organização e incorporá-lo a produtos, serviços e sistemas. Criar novos conhecimentos também não é apenas uma questão de aprender com os outros ou adquiri-los externamente.

Em uma Siderurgia, por tratar-se de um ambiente complexo, exige a combinação de técnicas e ferramentas para tratar a gestão do conhecimento. Conforme Small e Sage [3], o conhecimento que permanece na mente de uma pessoa é de pouca valia para uma organização a menos que seja compartilhado. O conhecimento siderúrgico não pode ser exclusivo do engenheiro, operador ou supervisor da planta, pois o turnover invoca riscos de perda na produção e riscos de segurança, devido à falta de conhecimento em operar corretamente uma planta industrial.

Este conhecimento (know-how) tácito deve perpetuar de forma explícita em manuais, procedimentos, treinamentos, normas, entre outros planos de capacitação profissional. O software de colaboração - Microsoft SharePoint - gerencia os documentos como manuais e procedimentos, e planilhas na Intranet da empresa, que pode ser acessada de qualquer estação com um browser instalado.

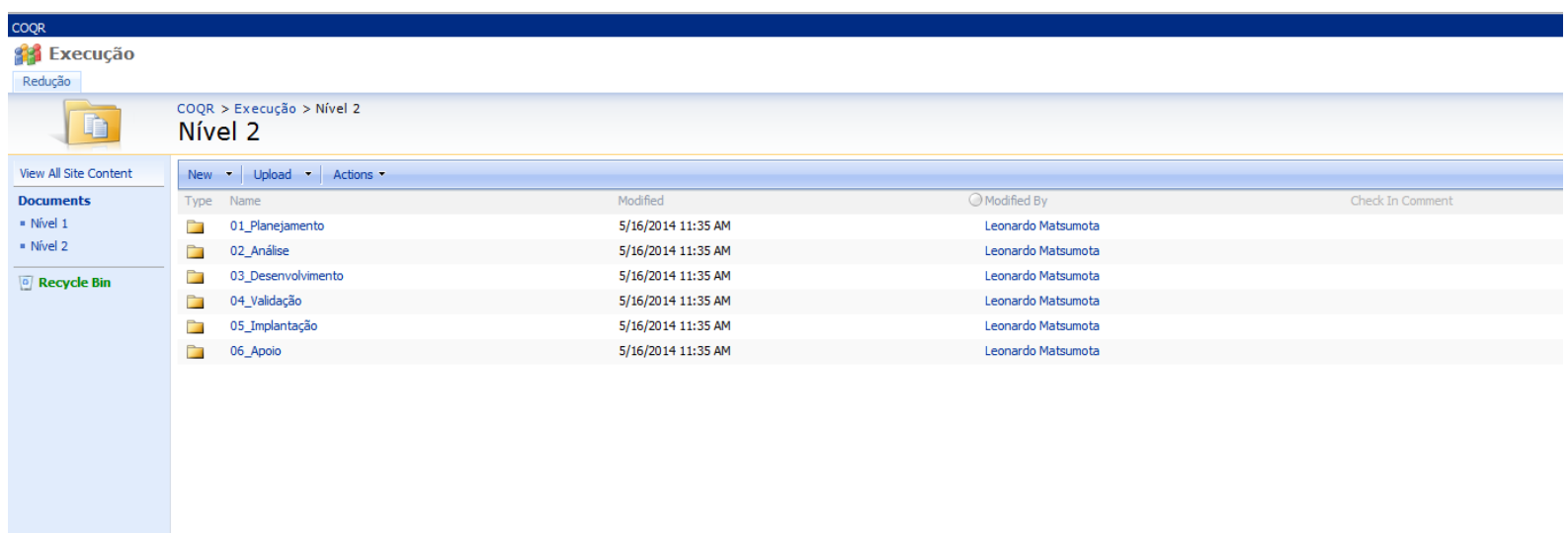

Figura 1 - Ambiente de colaboração para troca de conhecimento na empresa.

A troca de informação e os motivos que a influenciam dentro das empresas vem sendo discutida em trabalhos das áreas de Administração e Ciência da Informação Tsai et al. [4]; Ipê [5]; De Long e Fahyer [6]; Ives et al. [7]; Kim e Ju [8]; Keong e Al-Hawamdeh [9]; Lin [10]; Sabetzadeh e Tsui [11]; Yu et al. [12].

* Contribuição técnica ao $18^{\circ}$ Seminário de Automação e TI Industrial, 23 a 26 de setembro de 2014, São Paulo, SP, Brasil. 




Os fatores motivacionais do compartilhamento da informação, segundo McClelland [13] na Teoria das Necessidades Adquiridas, as necessidades humanas são aprendidas e adquiridas pelas pessoas ao longo de suas vidas. As três categorias da motivação humana propostas são: o relacionamento, a realização e o poder e o status como as necessidades básicas do indivíduo.

Criado o ambiente colaborativo, também foi necessário o levantamento dos eventos que compõem o processo de produção da Coqueria, conhecido por Operadores e Metalúrgicos. A execução dos serviços de instrumentação, elétrica, automação e TI possibilitou o registro destes eventos em banco de dados, que junto a dados operacionais e de produção, são apresentadas no sistema de gestão empresarial SAP - utilizado na empresa como figura abaixo.

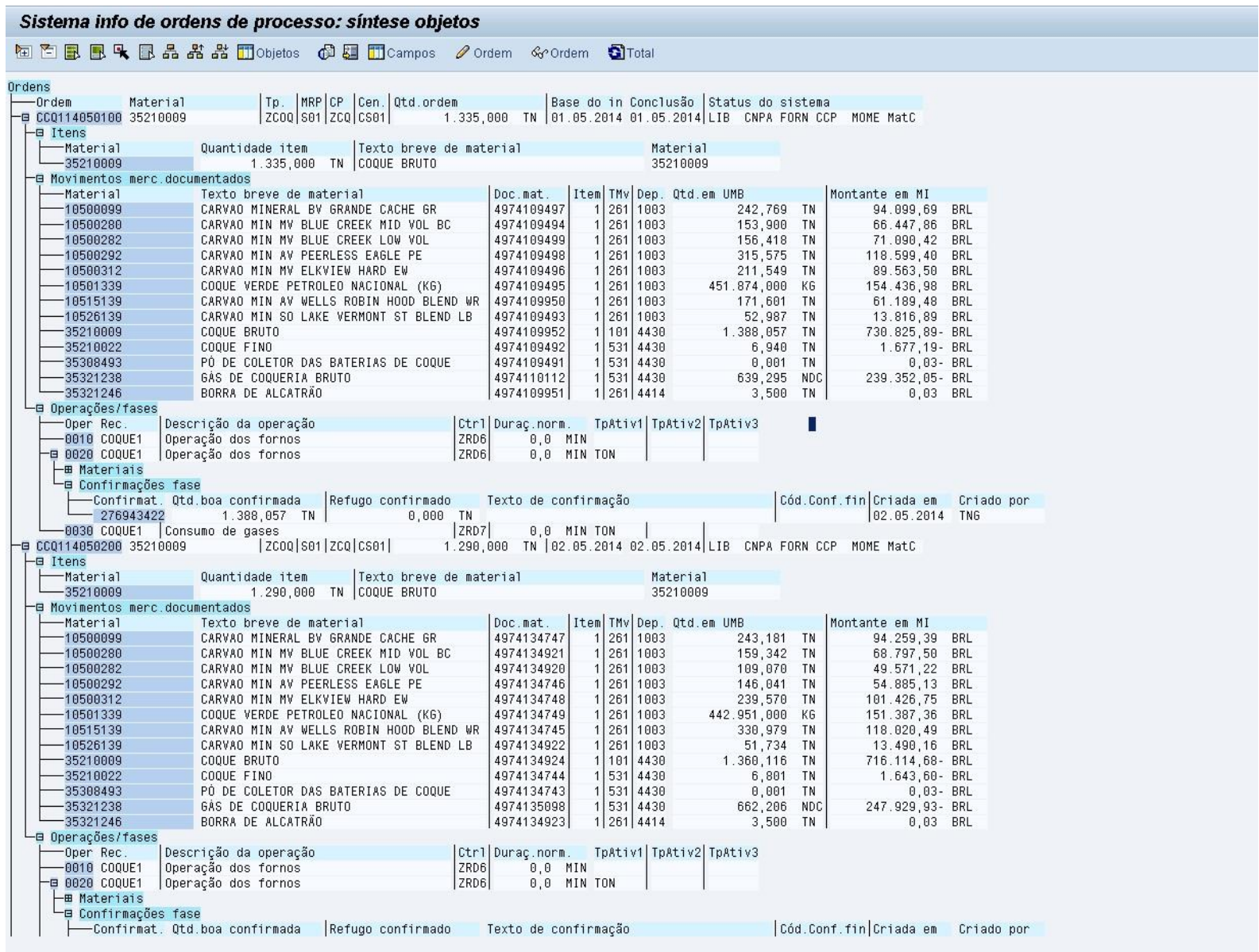

Figura 2 - Relatório dos dados de consumo e produção no Sistema Corporativo - SAP.

No entendimento de lógicas complexas como nas áreas de TI e Automação, a programação de controladores e aplicações (softwares) são fontes de conhecimento humano codificado para linguagem de máquina. $O$ desenvolvimento deve seguir metodologias e padrões para garantir a implantação de boas práticas e facilitar futuras manutenções nos sistemas. Lehman [14] diz que uma metodologia tem que atender a um ambiente complexo, imprevisível e de frequente mudança nos requisitos. Já Boehm e Turner [15], afirmam que as organizações precisam desenvolver se para o melhor equilíbrio entre agilidade e processos tradicionais que melhor se adaptam a sua situação.

Além da implantação dos sistemas, é importante um controle de versão com rastreamento das modificações realizadas para disponibilizar versões corretas aos

* Contribuição técnica ao $18^{\circ}$ Seminário de Automação e TI Industrial, 23 a 26 de setembro de 2014, São Paulo, SP, Brasil. 
colaboradores e também garantir uma recuperação imediata em caso de problema. Sommerville [16] define que as ferramentas de controle de versões têm a função de armazenar versões de componentes de sistema, de maneira que seja possível a construção dos sistemas a partir desses componentes e o acompanhamento dos releases do software para os clientes. O SourceSafe é uma ferramenta que gerencia as versões dos sistemas na empresa como figura abaixo.



Figura 3 - Controle de versão das aplicações com o Visual SourceSafe.

A aquisição dos dados (baseado nos eventos) feita pelas aplicações não é suficiente sem a utilização de tecnologias para o armazenamento e análise adequada sobre este grande volume de dados. O processo de ETL (extração, transformação e carga), figura a seguir, transforma estes dados em informação e assim gerar conhecimento para a empresa é extremamente importante por auxiliar na tomada de decisão e na área estratégica da organização. Os conceitos de Data Warehouse e Data Mining são imprescindíveis neste contexto de inteligência corporativa, pois permite estruturar os dados presentes em Banco de Dados e criar uma base de informações estratégicas para a empresa.

Segundo Kimball [17], o poder de soluções de Business Intelligence pode proporcionar a organização uma substancial vantagem competitiva em relação a seus competidores. Um pequeno investimento numa solução adequada de BI pode produzir retornos significativos.

* Contribuição técnica ao $18^{\circ}$ Seminário de Automação e TI Industrial, 23 a 26 de setembro de 2014, São Paulo, SP, Brasil. 

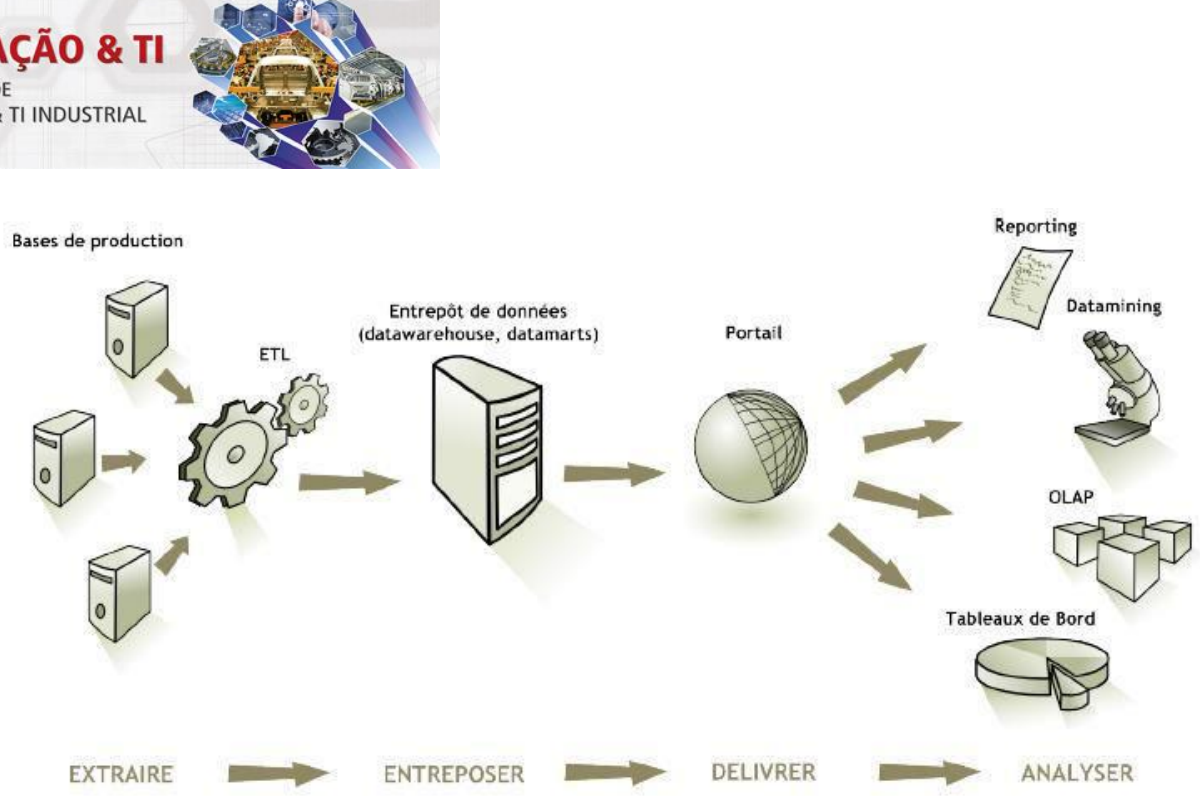

Figura 4 - Processo de ETL no ambiente de Data Warehouse [21].

\section{MATERIAIS E MÉTODOS}

De maneira geral, o processo de produção da Coqueria segue as seguintes etapas:

1. O recebimento da matéria prima, mistura de carvões, vinda do Pátio de Carvão e armazená-los em Silos para utilizar no processo de produção.

2. Realizar o Enfornamento do material nos Fornos.

3. Executar o processo de coqueificação, que consiste no aquecimento (ausência de ar), em média de 17 a 20 horas do material.

4. O material Coque é então Desenfornado (retirado do Forno) e passa por um processo de extinção (a base de água), onde é realizado o apagamento do Coque para estocagem ou transportado por correias até os Altos Fornos.

Seguindo as etapas no processo de produção citadas acima, o conhecimento siderúrgico de cada evento realizado por máquinas e equipamentos no chão de fábrica foi transferido para sistemas de automação e tecnologia, e assim obter informações confiáveis do processo.

O primeiro item foi obter a visualização de forma gráfica, os dados dos Níveis de Silos das Torres que armazenam a mistura de carvões que vão ser utilizados no processo. A instalação do sistema PIMS - da fabricante OSI em comunicação com o CLP da Rockwell SLC500 permite a aquisição e armazenamento de histórico dos valores dos níveis de cada Silo.

A figura abaixo demonstra o nível do Silo 1, 2 e 3 da Torre de Carvão do Grupo I de Baterias de Coque. Também é possível visualizar e analisar os dados do Grupo II que estão na tela inicial do processo da área da Redução.

* Contribuição técnica ao $18^{\circ}$ Seminário de Automação e TI Industrial, 23 a 26 de setembro de 2014, São Paulo, SP, Brasil. 


\section{AUTOMAÇÃO \& TI}

$18^{\circ}$ SEMINARIO DE

AUTOMAÇÃO \& TI INDUSTRIAL
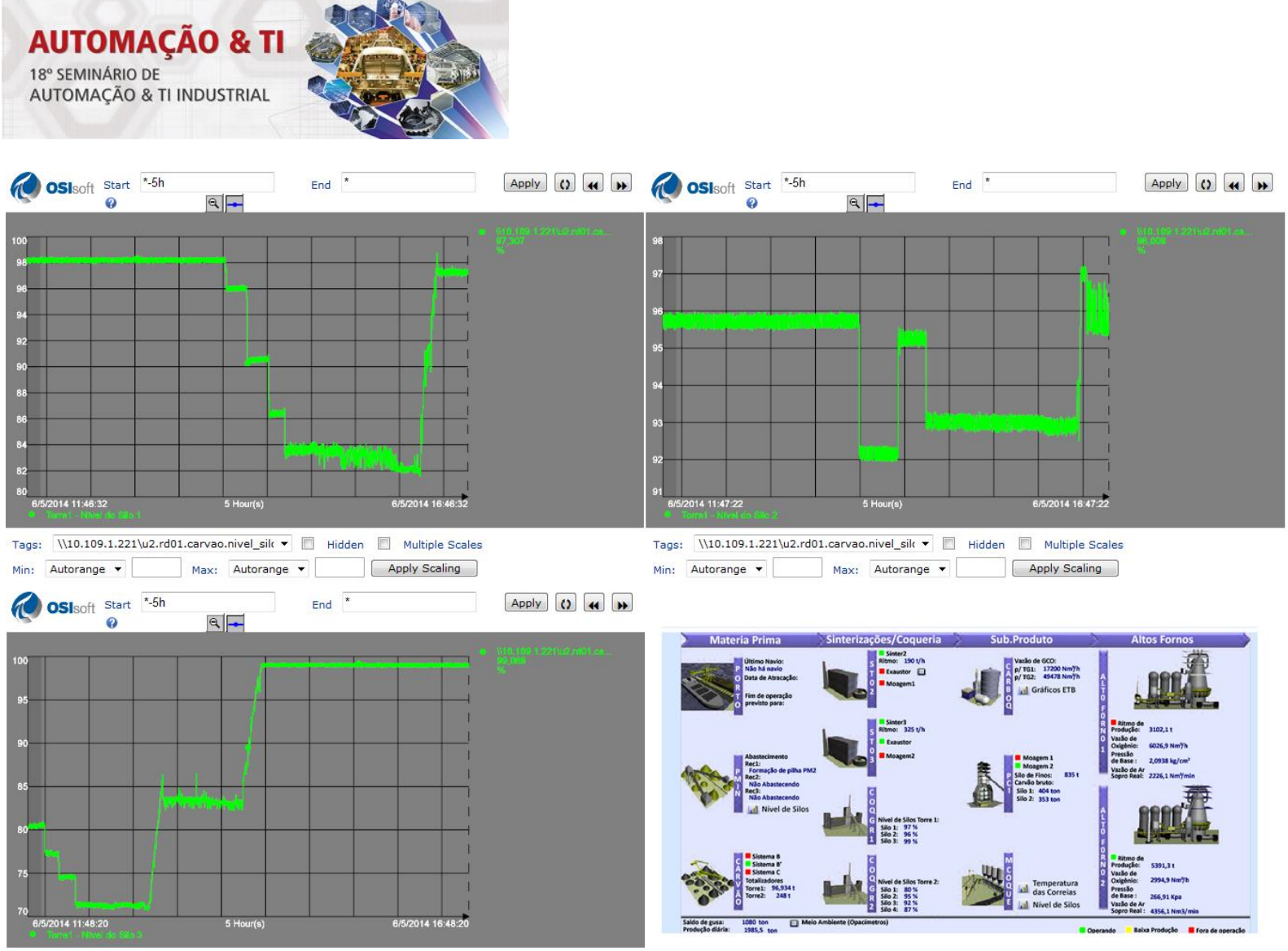

Tags: ||10.109.1.221\U2.rd01.carvao.nivel_silk - Hidden Multiple Scales

Min: Autorange $-\square$ Max: Autorange $-\square \square$ Apply Scaling

Figura 5 - Gráfico de nível de silo das Torres de Carvão que abastecem a Coqueria.

A etapa de Enfornamento é o próximo evento de controle para garantir a estabilidade operacional. De acordo com normas e procedimentos de operação, após carregar o material no Forno, por último deve ser feito um nivelamento para compactação do material. Este nivelamento é um sinal elétrico vindo da Máquina, que junto com o posicionamento (número do Forno que a Máquina se encontra), possibilita o registro destes acontecimentos, que caracterizam um novo Enfornamento.



Figura 6 - Gráfico de posição da Máquina e nivelamento dos Fornos - Etapa de Enfornamento.

* Contribuição técnica ao $18^{\circ}$ Seminário de Automação e TI Industrial, 23 a 26 de setembro de 2014, São Paulo, SP, Brasil. 
O processo de coqueificação requer um preciso controle da temperatura dos Fornos para determinação do tempo líquido de coqueificação. O gráfico apresenta a variação da temperatura do Forno 3 no período de uma semana. Podemos observar que a variação do processo é muito parecida na relação das variáveis de tempo e temperatura, e por isso o algoritmo do tempo líquido de coqueificação consegue estimar com precisão o momento certo de Desenfornamento do material.

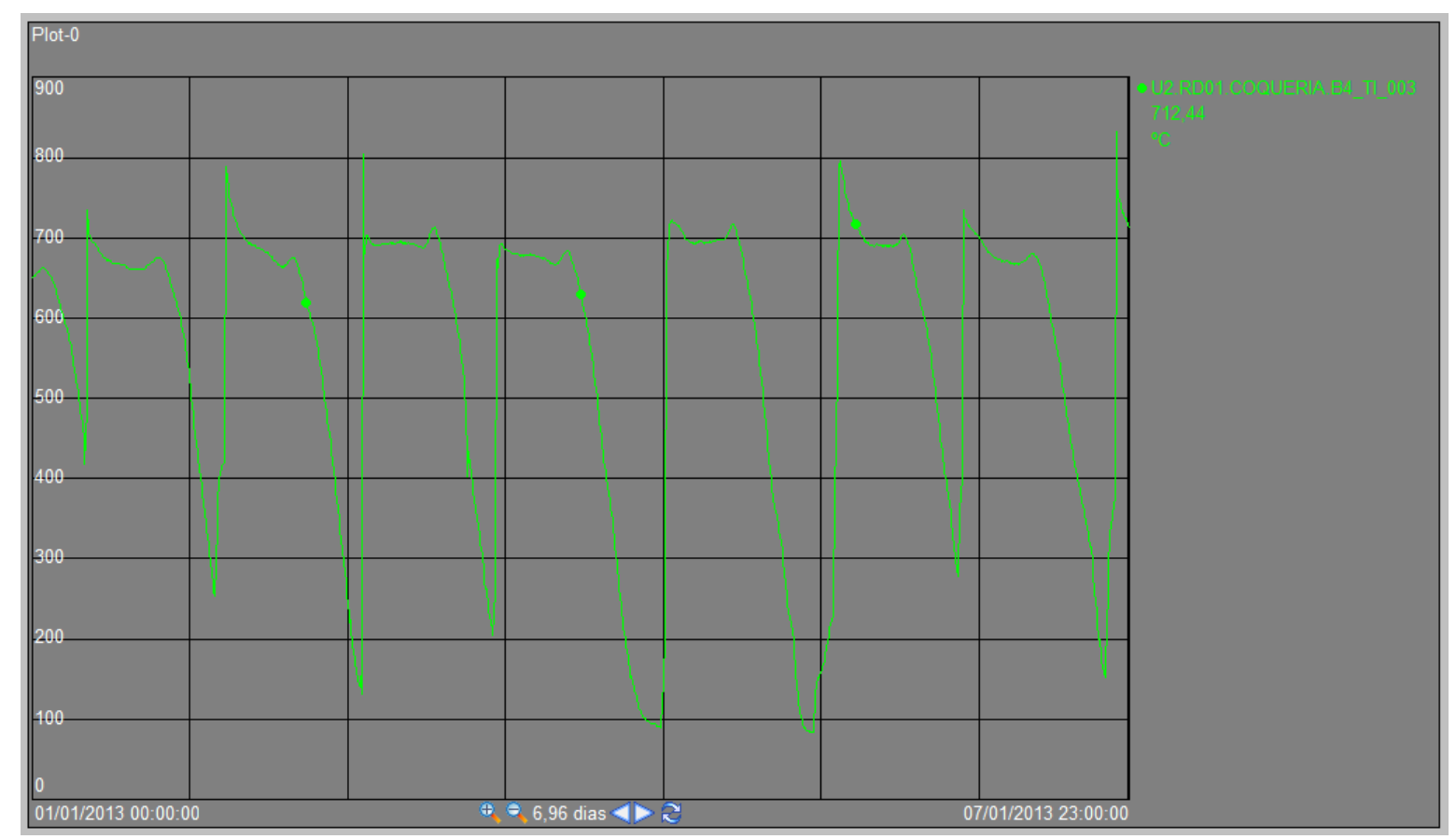

Figura 7 - Gráfico da temperatura do Forno, medida pelo Termopar instalado no Tubo de Ascensão.

Por fim, a última etapa é o Desenfornamento do Coque que está pronto para ser utilizado como combustível e agente redutor nos altos-fornos que provém o carbono adicionado ao ferro, gerando o aço.

Como vemos na figura a seguir, o processo de Desenfornamento do material acontece através da translação da barra de cremalheira da MD (Máquina Desenfornadora), acima de $20.000 \mathrm{~mm}$, que projeta o material ao CTC (Carro Transferidor de Coque) e CA (Carro Apagador) para finalizar o processo de produção. A medição da translação da cremalheira no interior do Forno mais o posicionamento da MD em relação ao Forno é o que possibilita conhecer o horário que ocorreu cada Desenfornamento.

Vale ressaltar que a sequência de Desenfornamento é realizada em séries de Fornos (Série de 1 a 5), e cada série compreende uma sequência de Fornos. A série 1 compreende os fornos $1,6,11,16 \ldots$, a série 2 com os fornos $2,7,12,17 \ldots$, isso porque existe um cálculo de ciclo de coqueificação que estima o número diário de Fornos objetivado pelo índice operacional. Em média, são 8 minutos entre o Desenfornamento dos Fornos.

* Contribuição técnica ao $18^{\circ}$ Seminário de Automação e TI Industrial, 23 a 26 de setembro de 2014, São Paulo, SP, Brasil. 


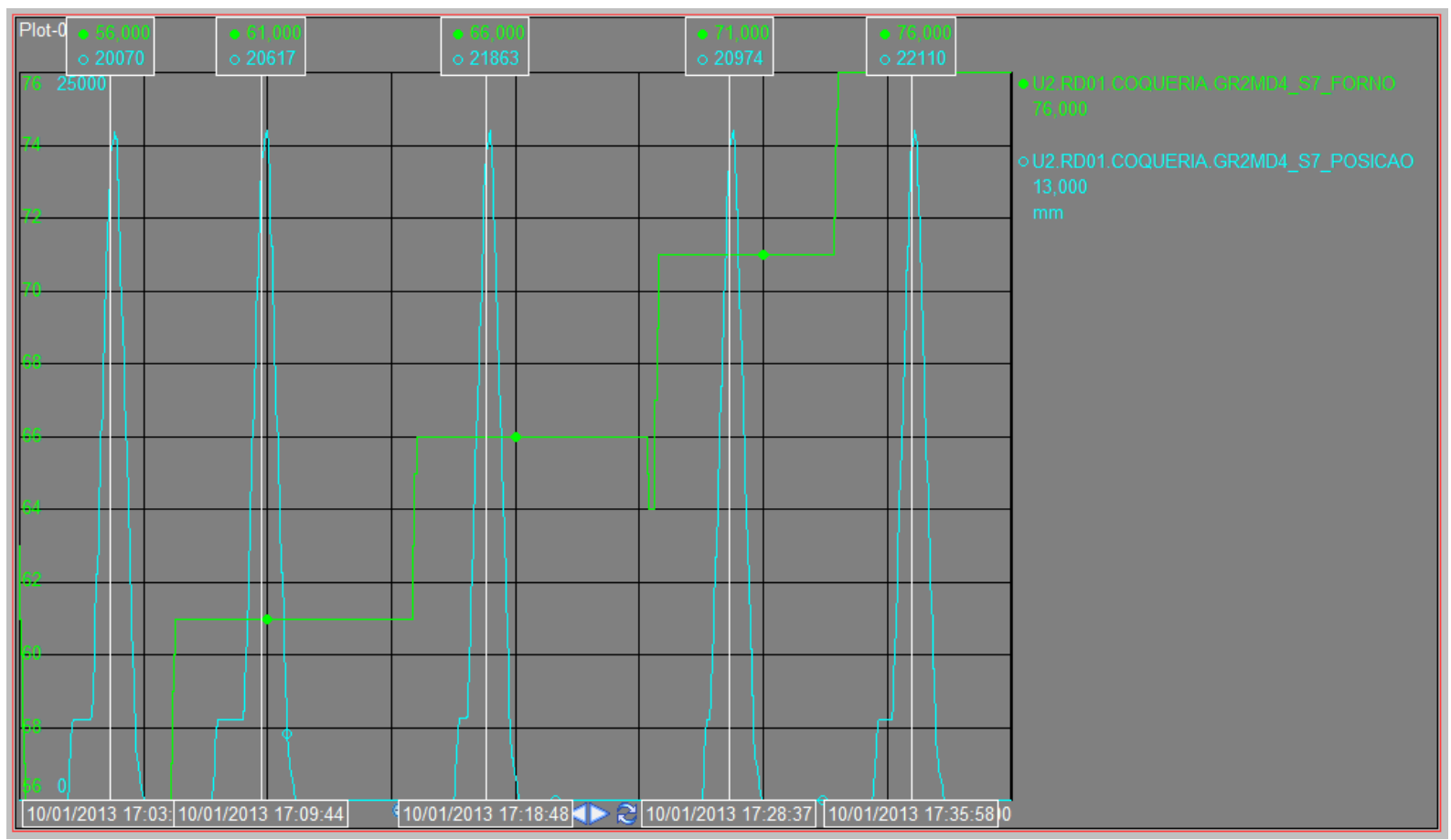

Figura 8 - Gráfico de posição da MD (Máquina Desenfornadora) e translação da barra cremalheira Etapa de Desenfornamento.

Após cumprir todas as etapas no processo de produção, vimos que todos os eventos foram identificados, monitorados e inseridos no sistema de automação da empresa. Desta forma, construímos uma base de dados com as principais informações inerentes ao processo operacional.

O Sistema Integrado das Coquerias foi desenvolvido em plataforma Web para apresentar relatórios com estas informações. Atualmente este sistema está integrado com o SAP, sistema de gestão empresarial utilizado na empresa e que possui módulos de diversas áreas como logística, materiais, financeiro, $\mathrm{RH}$, etc. e por isso, possibilita a empresa redimensionar a necessidade de produção do material, entre outras análises importantes que são feitas utilizando estes dados como fonte oficial de tomada de decisão.

A figura a seguir representa os módulos disponíveis no Sistema Integrado das Coquerias. Como exemplo, podemos ver um relatório de Desenfornamentos realizados em um dia de produção. Algumas informações são atualizadas manualmente pelos operadores, por ainda não estarem com medições automáticas confiáveis, como é o caso dos Pesos, Densidade, Umidade e Matéria Volátil.

Para acessar o sistema, foi compartilhado um link de acesso na Intranet da empresa e assim que acessado, o sistema efetua um controle de permissões e navegabilidade de acordo com perfil configurado para cada grupo de usuários: operadores, supervisores, suporte técnico, engenheiros e analista, gerentes e usuários que apenas visualizam páginas de acesso comum.

* Contribuição técnica ao $18^{\circ}$ Seminário de Automação e TI Industrial, 23 a 26 de setembro de 2014, São Paulo, SP, Brasil. 




Figura 9 - Sistema Integrado das Coquerias - Relatórios na Web.

A arquitetura do sistema de automação e tecnologia é composta dos seguintes itens:

- O CLP (Controlador Lógico Programável) Altus com uma CPU AL2003, cartões de comunicação Ethernet AL3145 e Profibus AL3406 é responsável pelo controle das temperaturas medidas no processo de coqueificação.

- O CLP Siemens Step7, responsável pelo controle das máquinas operacionais.

- Banco de Dados MS SQL Server 2008 para armazenamento dos dados.

- Visual Studio 2008 (Dot Net) e linguagem de programação C\# foram utilizados para a construção das aplicações que monitoram os eventos no CLP e fazem a gravação no banco de dados.

- Termopares com isolação mineral tipo $\mathrm{N}$ instalados no tubo de ascensão.

- Servidor OPC Kepware que permite a comunicação entre as aplicações e os controladores CLP.

- Sistema PIMS - PI da OSIsoft que permite o armazenamento dos dados e integração com ferramentas para gerar gráficos.

\footnotetext{
* Contribuição técnica ao $18^{\circ}$ Seminário de Automação e TI Industrial, 23 a 26 de setembro de 2014,
} São Paulo, SP, Brasil. 


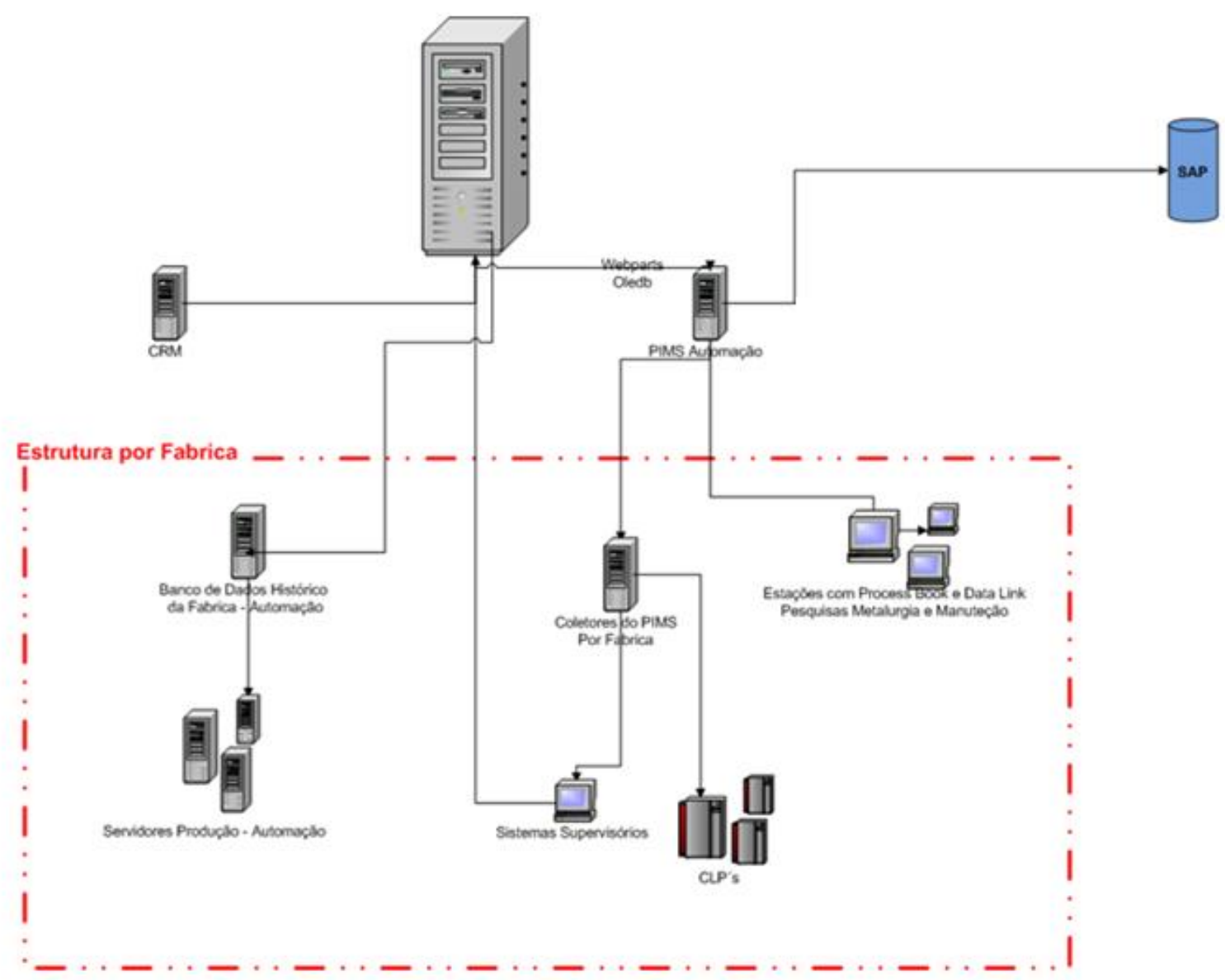

Figura 10 - Arquitetura do sistema de automação e tecnologia da área.

\section{RESULTADOS E DISCUSSÃO}

O tempo líquido de coqueificação é obtido por uma inspeção individual dos fornos, baseado em um conhecimento pessoal do Operador, através da visualização dos gases gerados pelo processo. Tanaka et alli [18], mostraram que o gás produzido pelo processo de coqueificação sofre uma variação característica da sua temperatura momentos antes do tempo líquido de coqueificação. Essa variação pode ser captada por termopares instalados nas partes curvas do tubo de ascensão dos fornos.

O modelo matemático que determina o ponto máximo de procura da Temperatura (TMax) depende da sequência de eventos citadas anteriormente. A TMax é procurada no intervalo entre 11 horas após o Enfornamento e a data da primeira temperatura abaixo da média. Com este ponto definido, calcula-se o tempo líquido de coqueificação previsto entre 16 horas e 20 horas.

* Contribuição técnica ao $18^{\circ}$ Seminário de Automação e TI Industrial, 23 a 26 de setembro de 2014, São Paulo, SP, Brasil. 

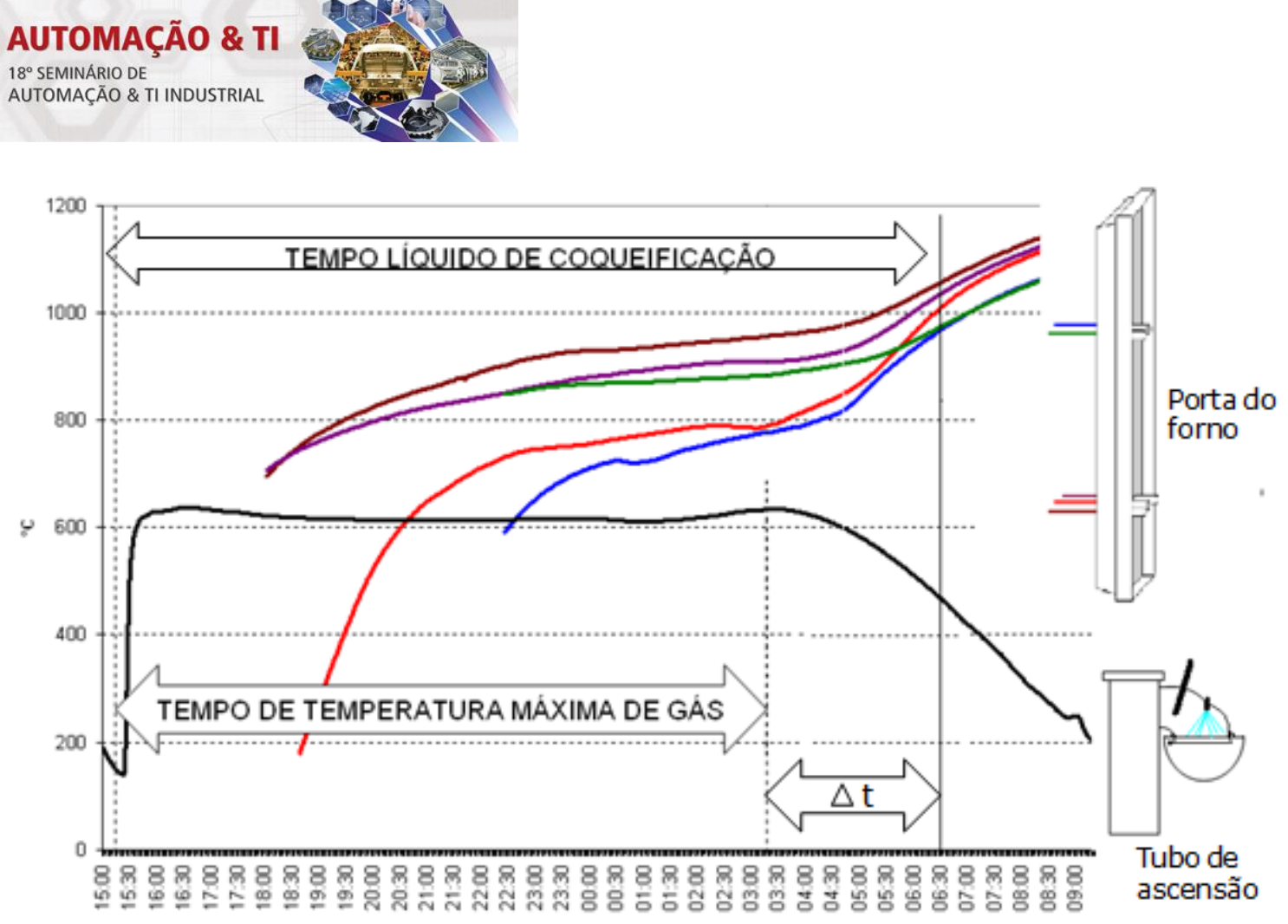

Figura 11 - Gráfico de temperatura do gás e da mistura de carvão enfornada ao longo da coqueificação.

Como relatado por Costa e Ramirez-Fernandez [19-20], a determinação do tempo líquido de coqueificação é feita no ponto onde as curvas das temperaturas dos vários termopares da carga começam a ter uma mesma inclinação, ou seja, o processo de coqueificação, endotérmico, já terminou e a carga do forno começa a ter elevação de temperatura na mesma velocidade. Para transferir essa indicação para o gráfico da temperatura dos gases produzidos, é determinado o delta t, ou seja, o tempo entre o momento de temperatura máxima atingida pelos gases produzidos e o ponto de tempo líquido determinado pelos termopares da carga. Esse delta $t$ é assumido como constante para todos os fornos e assim, através do ponto de temperatura máxima dos gases que podemos chamar de tmax, é possível determinar o tempo líquido de coqueificação.

\section{CONCLUSÃO}

O conhecimento siderúrgico é composto de inúmeras variáveis de alta complexidade, que necessitam de técnicas e ferramentas para obter sucesso no processo de transformação dos dados em informação, e do conhecimento tácito em explícito. Neste trabalho, vimos que o uso do ambiente colaborativo junto a técnicas de levantamento de requisitos contribuiu para o desenvolvimento de aplicações e construção de banco de dados que são utilizados como fonte oficial e base para tomadas de decisões na empresa. A construção do sistema da Coqueria, baseado no sistema de determinação do tempo líquido de coqueificação, necessitou do mapeamento dos eventos ocorridos durante o processo de produção, que antes eram conhecidos por Engenheiros, Operadores, Supervisores e colaboradores da empresa. No entanto, a implantação técnica destes eventos foi primordial para 0 desenvolvimento e sucesso na implantação do sistema. Entre os principais desafios gerados na área de automação e TI estão: a comunicação entre o CLP e as aplicações, que permitiu conhecer através de sinais elétricos o comportamento das máquinas que operam a planta (posicionamento, início e final de operação, etc.) para armazenar os eventos em banco de dados, e a comunicação entre os sistemas de

* Contribuição técnica ao $18^{\circ}$ Seminário de Automação e TI Industrial, 23 a 26 de setembro de 2014, São Paulo, SP, Brasil. 
automação e o sistema corporativo - SAP, que é realizado através do SAP XI (Exchange Infrastructure).

\section{Agradecimentos}

Ao Professor Dr. Oduvaldo Vendrametto, aos Mestres Luiz Claudio Costa e Nilson Magalhães Bueno e aos Engenheiros Jose Mauro Mendes, Ricardo Gomes Cupido e Leonardo Silva Costa. A todos das equipes de manutenção, automação, instrumentação e elétrica da empresa Usiminas.

\section{REFERÊNCIAS}

1 Tarapanoff K. (Org.). Inteligência organizacional e competitiva. Brasília: Universidade de Brasília, 2001.

2 Nonaka I, Takeuchi H. Criação de conhecimento na empresa. 9 ed. Rio de Janeiro: Campus, 1997.

3 Small C, Sage A. Knowledge management and knowledge sharing: A review. Information Knowledge Systems Management, 2006; 5(3): 153-169.

4 Tsai MT, Chen KS, Chien JL. The factors impact of knowledge sharing intentions: the theory of reasoned action perspective. Springer Science + Business Media B.V., online, mar. 2011. Disponível em <http://www.springerlink.com/content/g38077pw3066011q/>. Acesso em 10 maio. 2014.

5 IPE, M. Knowledge sharing organizations: a conceptual framework. Human Resource Development Review, v. 2, n. 4, p. 337-359, dez. 2003.

6 Long D, Fahey L. Diagnosing cultural barriers to knowledge management. Academy of Management Executive, v. 14, n 4, nov. 2000.

7 Ives W, Torrey B, Gordon C. Knowledge Sharing is human behavior. Knowledge Management: Classic and Contemporary Works, D. Morey, M. Maybury and B. Thuraisingham, eds, MIT Press, Cambridge MA, 2003.

8 Kim S, Ju B. An analysis of faculty perceptions: attitudes toward knowledge sharing and collaboration in an academic institution. Library \& Information Science Research, 2008; 30: 282-290.

9 Keong LC, Al-Hawamdeh S. Factors Impacting Knowledge Sharing. Journal of Information \& Knowledge Management, 2002; 1(1): 49-56.

10 LIN, Wen-Bao. The exploration factors of affecting knowledge sharing - The case of Taiwan's high-tech industry. Expert Systems with Applications, n. 35, p. 661-676, 2008.

11 Sabetzadeh F, Tsui E. Social motives polarity and its impact on knowledge sharing. VINE: The journal of information and knowledge management systems, 2011; 41(1): 76-88.

12 Yu TK, Lu LC Liu TF. Exploring factors that influence knowledge sharing behavior via weblogs. Computers in Human Behavior, v. 26, p. 32-41, 2010. Disponível em <http://www.researchgate.net/publication/200773103_Exploring_factors_that_influence knowledge_sharing_behavior_via_weblogs>. Acesso em 12 maio. 2014.

$13 \bar{M}$ CClelland $\bar{D}$ C. Human motivation. New York, NY: Cambridge University Press, 1987. $663 \mathrm{p}$.

14 Lehman M, Ramil J. Rules and Tools for Software Evolution Planning and Management. Annals of Software Engineering, 2001; 11(1): 15 - 44.

15 Boehm B, Turner R. (2004). Balancing agility and discipline: evaluating and integrating agile and plan-driven methods. In Proceedings of the 26th IEEE International Conference on Software Engineering (ICSE), pp. 718 - 719.

16 Sommerville I. Engenharia de Software. 6. Ed., São Paulo: Addison-Wesley, 2003.

17 Kimball R, Ross M. The Data Warehouse Tollkit. Tradução da $2^{2}$ edição original. Rio de Janeiro: Campus, 2002.

\footnotetext{
* Contribuição técnica ao $18^{\circ}$ Seminário de Automação e TI Industrial, 23 a 26 de setembro de 2014, São Paulo, SP, Brasil.
} 
18 Sadaki J, Tanaka K, Naganuma Y. Automatic coking control system. In: IEEE Conference on Control Applications, 2., Vancouver, 1993. Proceedings. Vancouver: IEEE, 1993; 2: 531-538.

19 Costa LC. Parâmetros do Controle de Processo de Coqueificação das Baterias de Fornos da Cosipa. 2008. 89p. Dissertação (Mestrado) Escola Politécnica da Universidade de São Paulo. Departamento de Engenharia de Sistemas Eletrônicos.

20 Costa LC, Ramirez-Fernadez FJ. Parameters for the cokemaking process control. In: III International Meeting on Ironmaking, 2008, São Luiz - MA. Annals 3rd International Meeting on Iromaking. São Paulo: ABM.

21 Developpez.com [acesso em 6/5/2014]. Disponível em: http://businessintelligence.developpez.com/. 